\title{
Asteroseismology of B stars
}

\author{
A. Thoul ${ }^{1,2}$ \\ ${ }^{1}$ Université de Liège, 17 allée du 6 août, Bât B5c, B-4000 Liège, Belgium
}

\begin{abstract}
Numerous B stars on the main sequence are found to be variable. These stars have a relatively simple structure, and yet they present some very interesting challenges. It is important to understand these stars before we can expect to be able to understand stars which have a more complicated internal structure.

Asteroseismology of B stars has made considerable progress in the last few years, thanks to the observations obtained from large multisite campaigns, and it is now possible to determine global parameters for these stars such as their masses, ages, metallicities, with very high accuracy. Many variable B stars are also observed with the CoRoT space mission, and statistical studies may become possible in the near future.

Detailed studies of the oscillation spectra of $\beta$ Cephei stars have already allowed to put some limits on the overshooting parameter, and this overshooting parameter is found to vary from one star to the next. Some $\beta$ Cephei stars are found to present differential rotation in their envelopes, while others are compatible with solid body rotation. Some B stars present nitrogen enhancement, even though they are very slow rotators. The instability strips of B stars differ depending on the composition and the opacity tables adopted. Microscopic diffusion and radiative accelerations could produce an accumulation of iron-group elements in some layers of these stars. Hybrid pulsators, showing both $\beta$ Cephei and SPB pulsations have been observed. Some of the best observed stars have pulsation spectra which still cannot be reproduced by modelling.

Here I review the present status of the observations and of the modelling of $\beta$ Cephei stars, emphasizing both the successes reached and the questions and problems which remain open today.
\end{abstract}

Individual Objects: 16 Lac, HD 129929, $\nu$ Eri, $\theta$ Oph, 12 Lac

\section{Introduction}

Classical stellar observables, such as the effective temperature, the surface gravity, the luminosity, and the metallicity, are not sensitive to the internal structure of the stars, and by themselves do not give enough information for the determination of the basic stellar parameters. However, pulsation frequencies are very sensitive to the details of the internal structure. By comparing the oscillation spectra to those computed from theoretical models, it is possible to obtain accurate global stellar parameters, such as stellar masses, radii, temperatures, luminosities, metallicities, but also to improve the physics used in the theoretical models, such as the physics of convection, rotational processes, mixing, equation of state, nuclear reaction rates, opacities, microscopic diffusion, radiative accelerations, mass loss, and magnetism.

\footnotetext{
${ }^{2}$ Chercheur Qualifié FNRS
} 
Pulsating main sequence B stars can be classified as $\beta$ Cephei, SPB or Be stars. They have a relatively simple internal structure, with a massive convective core and a radiative envelope. The pulsations in these stars are excited by the $\kappa$ mechanism due to the iron opacity bump at $200000 \mathrm{~K}$. These are long-lived intrinsically unstable modes. Long-term multisite photometric and spectroscopic campaigns are needed to resolve the oscillation frequencies of these stars. Several such campaigns have been organized to observe single $\beta$ Cephei stars; detailed asteroseismic studies of these stars have been performed, and the results are described in the next section. Both photometric and spectroscopic observations are needed because they sample the same pulsations, but with different sensitivity to the degree and azimuthal order of the modes. Photometric observations measure weighted averages of the pulsation amplitude over the $\tau=2 / 3$ surface, so they are mostly sensitive to low-degree modes. Doppler observations measure the projection of the velocity over the line of sight, and are therefore slightly more sensitive to modes of moderate degree $I$. Photometry is commonly used to observe pulsations in main sequence B stars. Multicolour photometry and spectroscopy are however essential, because, as we will show later, it is necessary to identify the modes to do useful asteroseismology with these stars. The amplitude and phase behaviour of an oscillation mode are different in different filters, so that the degree $I$ can be determined through multi-colour photometry. Line-profile variations are determined by parameters including the degrees $I$ and azimuthal orders $m$ of all the pulsational velocities.

Pulsating Be stars are fast rotators with emission lines. Asteroseismology of these complicated stars is still in its infancy. SPB stars have masses between 4 and $7 \mathrm{M}_{\odot}$. They pulsate in the asymptotic regime of high-order $\mathrm{g}$ modes with periods of 0.5 to 5 days. This is a serious observational difficulty, and very long multisite campaigns are needed. At this time, much fewer modes are detected than expected, and they cannot be identified due to their low amplitudes. The asteroseismology of SPB stars is therefore also still in its infancy. $\beta$ Cephei stars are more massive; their masses range between 8 and $18 \mathrm{M}_{\odot}$. They are mostly slow rotators, and have a rather sparse spectrum of low-degree, low-order p, g and mixed modes, with periods of 2 to 8 hours. In $\beta$ Cephei stars, the individual frequencies probe different layers of the star, and therefore, each frequency in the spectrum gives independent information about the stellar interior.

\section{Detailed asteroseismic studies of $\beta$ Cephei stars}

Asteroseismic studies of $\beta$ Cephei stars consist of performing direct frequency fitting of axisymmetric modes. This can provide accurate determinations of basic stellar parameters, constraints on the core overshooting parameters and on the metallicity, or it can also lead to inconsistencies, i.e., information on missing or incorrect physics in the stellar models. The multiplets are then analyzed, and the splittings are used to get information on the rotation rate in the stellar envelope.

Main-sequence B stars are modelled using "standard" stellar evolution codes. The modelling parameters are typically the stellar mass $M$, the initial hydrogen mass fraction $X$, the metallicity $Z$, and the overshooting parameter $\alpha_{o v}$, which is a measure of the extent of the mixed region at the boundary of the convective core. Several different prescriptions can be used for some of the physics needed in the stellar models, such as the theory of convection, opacity tables, gravitational settling and radiative accelerations, the initial chemical abundances, rotation ...

Here we give the main results which were obtained from detailed modelling of several $\beta$ Cephei stars, the successes and problems encountered, and conclusions that were reached. 


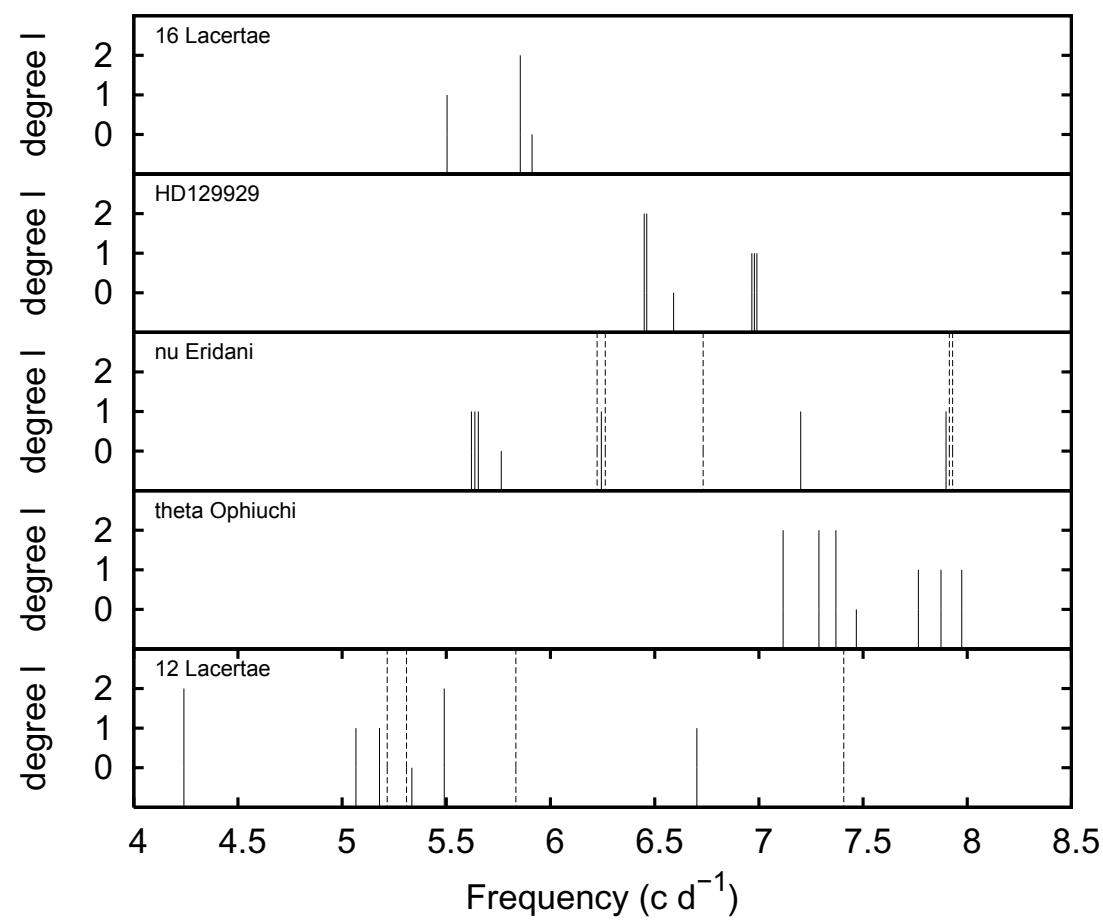

Figure 1: Observed independent frequencies in the five $\beta$ Cephei stars 16 Lac, HD 129929, $\nu$ Eri, $\theta$ Oph and 12 Lac. Solid lines are used for identified modes, while dashed lines are used for modes with no definite identification.

\section{Lacertae}

16 Lacertae is a spectroscopic binary and eclipsing variable. Its luminosity and effective temperature are constrained well. Three oscillation frequencies have been detected in this $\beta$ Cephei star. The degrees of these three modes of oscillation have been identified through spectroscopic and photometric mode identification methods (Chapellier et al. 1995, Dziembowski \& Jerzykiewicz 1996, Aerts et al. 2003, Thoul et al. 2003). Two of the modes are axisymmetric, but there are no multiplets. The frequencies of the three main modes of pulsation observed in $16 \mathrm{Lac}$ are $f_{1}=5.9112 \mathrm{~cd}^{-1}$, which is a radial mode, $f_{2}=5.8551 \mathrm{~cd}^{-1}$, which is an $I=2$ axisymmetric mode, and $f_{3}=5.5033 \mathrm{~cd}^{-1}$, which is an $I=1$ mode. They are represented in Fig. 1.

Evolutionary sequences have been calculated with the stellar evolution code CLES (Scuflaire et al. 2008a) and oscillation frequencies for each model were calculated using the adiabatic stellar oscillations code OSCL (Scuflaire et al. 2008b). For each set of model parameters $\left(M, X, Z, \alpha_{o v}\right)$, fitting the radial mode gives the age of the star, or, equivalently, its effective temperature $T_{\text {eff }}$. For a given set of model parameters $\left(X, Z, \alpha_{o v}\right)$, fitting the second axisymmetric frequency fixes the age and the mass $M$. It is interesting to note that $T_{\text {eff }}$ and $\log g$ are not imposed in the models; their values result from the models which fit the observed frequencies. The observed error box is however extremely useful to constrain the possible ranges for the stellar parameters. 
The mode with frequency $f_{1}$ was identified by the modelling as an $I=0 \mathrm{p}_{1}$ mode, $f_{2}$ as an $I=2 \mathrm{~g}_{1}$ mode and $f_{3}$ as an $I=1 \mathrm{~g}_{1}$ mode; this last one is in fact a mixed mode. The models were performed for only two values of $\alpha_{o v}$, namely 0 and 0.2 . Very precise values were obtained for the stellar mass, metallicity, effective temperature, luminosity and age (Thoul et al. 2003).

\section{HD 129929}

The star HD 129929 has been observed for over 20 years. Six independent pulsation frequencies were observed and identified, including one radial mode, one complete triplet and two members of a quintuplet (Aerts et al. 2004). Detailed asteroseismology of this star is possible since two axisymmetric modes are observed, and information about the internal rotation rate can be obtained from the two multiplets (Aerts et al. 2003b, Dupret et al. 2004).

The frequencies of the six independent modes of pulsation observed in HD 129929 are $f_{1}=6.461699 \mathrm{~cd}^{-1}, f_{2}=6.978305 \mathrm{~cd}^{-1}, f_{3}=6.449590 \mathrm{~cd}^{-1}, f_{4}=6.990431 \mathrm{~cd}^{-1}$, $f_{5}=6.590940 \mathrm{~cd}^{-1}$, and $f_{6}=6.966172 \mathrm{~cd}^{-1}$. $f_{5}$ is a radial mode, $f_{2}, f_{4}$ and $f_{6}$ form an $I=1$ triplet, and $f_{1}$ and $f_{3}$ are two members of a quintuplet. They are represented in Fig. 1.

Seismic modelling of HD 129929 was performed using CLES and OSCL. The radial mode is identified by the modelling as a $\mathrm{p}_{1}$ mode, the central frequency of the triplet corresponds to a $\mathrm{p}_{1}$ mode, and $f_{1}$ and $f_{3}$ are confirmed to be members of an $I=2 \mathrm{~g}_{1}$ quintuplet. The excitation of the modes was analyzed using MAD, a non-adiabatic oscillation code (Dupret et al. 2003). Constraints on the metallicity were obtained by requiring good compatibility between the observed and calculated amplitude ratios, and that the observed modes must be excited; this excluded solutions with no overshooting. In addition, in order to find solutions that fit the quintuplet, solutions with an overshooting larger than 0.15 had to be excluded also. Very precise values were obtained for the effective temperature, luminosity, mass, and age of the star.

The rotational splitting integrals of the two multiplets have amplitude peaks at different depths in the star; the two multiplets therefore give information about the rotation rates at different layers inside the star. It was possible to rule out rigid rotation in the star's envelope (Aerts et al. 2003b, Dupret et al. 2004).

\section{$\nu$ Eridani}

Two large multisite photometric campaigns and one large multisite spectroscopic campaign were dedicated to the star $\nu$ Eridani. From these campaigns, twelve independent frequencies were obtained and seven of these frequencies were identified (Handler et al. 2004, Aerts et al. 2004b, De Ridder et al. 2004, Jerzykiewicz et al. 2005). The frequencies of $\nu$ Eri are $f_{1}=5.7632828 \mathrm{~cd}^{-1}, f_{2}=5.6538767 \mathrm{~cd}^{-1}, f_{3}=5.6200186 \mathrm{~cd}^{-1}, f_{4}=5.6372470 \mathrm{~cd}^{-1}$, $f_{5}=7.898200 \mathrm{~cd}^{-1}, f_{6}=6.243847 \mathrm{~cd}^{-1}, f_{7}=6.262917 \mathrm{~cd}^{-1}, f_{8}=7.20090 \mathrm{~cd}^{-1}$, $f_{9}=7.91383 \mathrm{~cd}^{-1}, f_{10}=7.92992 \mathrm{~cd}^{-1}, f_{11}=6.73223 \mathrm{~cd}^{-1}, f_{12}=6.22360 \mathrm{~cd}^{-1}$. In addition, two low-frequency modes typical of SPB pulsations were observed, with frequencies $f_{A}=0.432786 \mathrm{~cd}^{-1}$ and $f_{B}=0.61440 \mathrm{~cd}^{-1} . \nu$ Eri has been called a "hybrid $\beta$ Cephei/SPB pulsator". The frequency $f_{1}$ is identified as a radial mode, the frequencies $f_{2}, f_{3}$ and $f_{4}$ form an $I=1$ triplet; $f_{5}, f_{6}, f_{8}$ are identified as $I=1$ modes. They are shown in Fig. 1 .

Detailed modelling of this star has been performed by several groups (Ausseloos et al. 2004, Pamyatnykh et al. 2004, Dziembowski \& Pamyatnykh 2008). Solutions were found that reproduced the observed frequencies of $\nu$ Eri, but those frequencies were not excited. Some authors suggested that the modes could be excited if iron accumulates in the excitation region due to microscopic diffusion. Others suggested that non-standard stellar models should be considered. In both cases, some of the high-frequency modes were excited and the two low-frequency modes were still stable. Recently, changes in solar abundances were reported 
(Asplund et al. 2005), and new opacity tables made available (Badnell et al. 2005). It was shown that these new abundances and opacities displace the blue edge of the instability strips of $\beta$ Cephei and SPB stars towards higher temperatures and increase considerably the potential number of hybrid pulsators (Miglio et al. 2007). Using these new abundances and opacity tables in the modelling of $\nu$ Eri, solutions were found where all high frequency modes (except for the highest one) are excited, and where a range of g modes is excited, which includes one of the two low-frequency modes observed (Dziembowski \& Pamyatnykh 2008).

Modelling of $\nu$ Eri was performed using the stellar evolution code CLES (Scuflaire et al. 2008a) with the Asplund et al. (2005) abundances and the new OP opacities (Badnell et al. 2005), and the oscillation frequencies were calculated using the oscillation code OSCL (Scuflaire et al. 2008b), for two values of the hydrogen mass fraction, namely $X=0.72$ and $X=0.70$. Models are found which fit exactly the four axisymmetric modes: $f_{1}$ is a radial $\mathrm{p}_{1}$ mode, $f_{4}$ is an $I=1 \mathrm{~g}_{1}$ mode, $f_{6}$ is an $I=1 \mathrm{p}_{1}$ mode, and $f_{8}$ is an $I=1 \mathrm{p}_{2}$ mode. The mass of the star is $M=9.0 \pm 0.1 \mathrm{M}_{\odot}$, the overshooting parameter is $\alpha_{o v}=0.22$, and the metallicity $Z=0.021 \pm 0.001$. The high frequency modes $f_{8}, f_{5}, f_{9}$ and $f_{10}$ are not excited. Low-frequency high-order $\mathrm{g}$ modes are excited in the range $0.55-0.91 \mathrm{~cd}^{-1}$. In these models, $f_{B}$ is excited, but not $f_{A}$.

The problems encountered in modelling the $\beta$ Cephei star $\nu$ Eri have been mostly solved when the new solar abundance and the new OP opacity tables were used. Some problems remain however, since the highest frequency observed and one of the low frequency modes are still not excited.

\section{$\theta$ Ophiuchi}

Photometric and spectroscopic campaigns were dedicated to the $\beta$ Cephei star $\theta$ Ophiuchi (Handler et al. 2005, Briquet et al. 2005). Seven independent frequencies were observed in a narrow frequency range; they were all identified, and included one radial mode, $f_{3}=7.4677 \mathrm{~cd}^{-1}$, one triplet, $f_{4}=7.7659 \mathrm{~cd}^{-1}, f_{6}=7.8742 \mathrm{~cd}^{-1}, f_{7}=7.9734 \mathrm{~cd}^{-1}$; and three members of a quintuplet, $f_{1}=7.11600 \mathrm{~cd}^{-1}, f_{5}=7.2881 \mathrm{~cd}^{-1}, f_{2}=7.3697 \mathrm{~cd}^{-1}$. They are represented in Fig. 1.

Detailed seismic modelling was performed for this $\beta$ Cephei star using the codes CLES and OSCL, with the new solar abundances, the increased abundance of neon from Cunha et al. (2006), and the new OP opacities, for two values of the hydrogen mass fraction, namely $X=0.71$ and $X=0.72$, and for metallicities $Z=[0.009,0.015]$. Models are chosen such that the two axisymmetric modes are reproduced; the quintuplet is used to obtain additional constraints. The models have a high overshooting parameter, $\alpha_{o v}=0.44 \pm 0.07$ and a mass $M=8.2 \pm 0.3 \mathrm{M}_{\odot}$. All solutions are inside the photometric error box, but outside the $2 \sigma$ spectroscopic error box. All observed modes are excited. The unequal splittings of the triplet can be reproduced by taking into account second order effects of rotation (Briquet et al. 2007).

\section{Lacertae}

12 Lacertae is probably the most puzzling $\beta$ Cephei star observed so far. It has been full of surprises. It had already been observed as a variable star in 1915 (Young 1915), with frequency $1 / P=5.17896 \mathrm{~cd}^{-1}$, a value very close to the present value of its main mode of oscillation, $5.179034 \mathrm{~cd}^{-1}$. It was already observed as a multiperiodic variable star in 1957 (Abrami 1957). Two frequencies of oscillation have been known with great accuracy since 1961 (Opalski \& Ciurla 1961): $f_{1}=5.17897 \mathrm{~cd}^{-1}$ and $f_{2}=5.06666 \mathrm{~cd}^{-1}$. The present values for these two frequencies are $5.179034 \mathrm{~cd}^{-1}$ and $5.066346 \mathrm{~cd}^{-1}$. In 1978 , six frequencies were determined, including an $I=3$ equidistant triplet (Jerzykiewicz 1978, Jarzebowski et al. 1980). The modes of the triplet were later contrarily identified as $I=2$ (Smith 
1980). Attempts were made in 1994 to discriminate between the different identifications using the moment method, without success (Mathias 1994). Identification was attempted through modelling in 1999 (Dziembowski \& Jerzykiewicz 1999), again with no result. In fact modelling of 12 Lac seems to be problematic. In 2006, a large multisite campaign was dedicated to $12 \mathrm{Lac}$. Ten independent frequencies were observed, six were identified, with a big surprise: The three frequencies in the very equidistant triplet are identified with three different values of the degree $I$ : they do not form a triplet after all (Handler et al. 2006)! The frequencies observed are $f_{1}=5.179034 \mathrm{~cd}^{-1}, f_{2}=5.066346 \mathrm{~cd}^{-1}$, which are both identified as $I=1$ modes, $f_{3}=5.490167 \mathrm{~cd}^{-1}$, identified as an $I=2$ mode, $f_{4}=5.334357 \mathrm{~cd}^{-1}$, a radial mode, $f_{5}=4.24062 \mathrm{~cd}^{-1}$, identified as $I=2, f_{6}=7.40705 \mathrm{~cd}^{-1}, f_{7}=5.30912 \mathrm{~cd}^{-1}$, $f_{8}=5.2162 \mathrm{~cd}^{-1}, f_{9}=6.7023 \mathrm{~cd}^{-1}$, identified as an $I=1$ mode, $f_{10}=5.8341 \mathrm{~cd}^{-1}$, and a low-frequency mode of frequency $f_{A}=0.35529 \mathrm{~cd}^{-1}$ (Desmet et al. 2007). These frequencies are represented in Fig. 1, except for the low-frequency g mode.

A first attempt at modelling $12 \mathrm{Lac}$ with these new identifications was performed by Dziembowski \& Pamyatnykh (2008). They assumed a hydrogen mass fraction of $X=0.70$, a metallicity $Z=0.015$, a chemical abundance determined by the solar composition of Asplund et al. (2004), and they used the new OP opacities. They used the following error boxes for the position in the HR diagram: $\log T_{\text {eff }}=[4.355,4.395], \log L=[4.0,4.35]$. Their calculations were performed without overshooting. They fitted the mode corresponding to $f_{4}$ with the radial fundamental mode, and $f_{2}$ with an $I=1 \mathrm{~g}_{1}$ mode. They excluded any other possible identification. Desmet et al. (2009) recently performed a detailed modelling of 12 Lac, using CLES with the newest solar abundances (Asplund et al. 2005), OP opacities, $X=0.72, Z=0.015$, and an error box in the HR diagram given by $\log T_{\text {eff }}=4.389 \pm 0.018$, $\log g=3.65 \pm 0.15$. Using the identification of $f_{9}$, they rule out $f_{4}$ as the radial fundamental, and identify this mode as the first overtone. The range of theoretically predicted unstable modes does not cover the whole range of observed modes; the two highest frequencies are not excited, and the SPB-type pulsation mode $f_{A}$ is not excited.

\section{Conclusions}

Detailed asteroseismic studies of a few $\beta$ Cephei stars have been very successful. They have shown that very precise determinations of basic stellar parameters such as the mass, luminosity, effective temperature, metallicity, and age can be obtained. In addition, precise values of the overshooting parameter can be derived. So far, $\alpha_{o v}$ takes values from 0 to 0.4 and is found to vary from one star to another. In some stars, zero overshooting can be ruled out. Evidence for non-rigid rotation in the stellar envelope has been found for at least one $\beta$ Cephei star, namely HD 129929. The modelling of $\nu$ Eri has been problematic. Using the new solar abundances and the new OP opacity tables in the models have helped to reduce considerably the discrepancy between the observations and the theoretical models, but the problem is not completely solved. The long and complicated story of the mode identification of the frequencies observed in 12 Lacertae has shown that good and reliable mode identification, using both spectroscopic observations and multicolour photometry, is essential before any useful detailed modelling can be performed.

The observational challenges are therefore to obtain very good frequency determinations, which is particularly difficult for SPB stars due to the long periods of the high-order $g$ modes, and reliable mode identifications, which are sometimes very tricky since multiplets due to rotational splittings can overlap with the spectrum of axisymmetric frequencies. A good determination of basic stellar parameters $\left(T_{\text {eff }}, \log g,[M / H]\right)$ is very helpful to rule out some of the models and reduce the parameter space. Therefore, even though a huge amount of photometric data will soon be available from space observations, it is imperative to perform ground-based follow-up observations with multicolour photometry and high-resolution spectroscopy. 
The main theoretical challenges at this time are to explain the range of excited modes in $\beta$ Cephei stars, the presence of $\beta$ Cephei and SPB stars in low metallicity environments, and the existence of hybrid SPB $\beta$ Cephei pulsators. Asteroseismic studies of B type pulsators will give constraints which will lead to improvements in the physics used in the models, such as the modelling of rotation, mixing, convection, ... As the study of $\nu$ Eri has proven, reliable opacity tables as well as correct initial chemical compositions are crucial.

Acknowledgments. AT is grateful to the organizers of the Symposium for inviting her to give a review talk on this topic, and to HELAS for providing financial support.

\section{References}

Abrami, A. 1957, Nature, 180, 1112

Aerts, C., Lehmann, H., Briquet, M., et al. 2003, A\&A, 399, 639

Aerts, C., Thoul, A., Daszynska, J., et al. 2003b, Science, 5627, 1926

Aerts, C., Waelkens, C., Daszynska-Daszkiewicz, J., et al. 2004, A\&A, 415, 241

Aerts, C., De Cat, P., Handler, G., et al. 2004b, MNRAS, 347, 463

Asplund, M., Grevesse, N., Sauval, A. J., et al. 2004, A\&A, 417, 751

Asplund, M., Grevesse, N., Sauval, A. J., et al. 2005, A\&A, 431, 693

Ausseloos, M., Scuflaire, R., Thoul, A., \& Aerts, C. 2004, MNRAS, 355, 352

Badnell, N. R., Bautista, M. A., Butler, K., et al. 2005, MNRAS, 360, 458

Briquet, M., Lefever, K., Uytterhoeven, K., \& Aerts, C. 2005, MNRAS, 362, 619

Briquet, M., Morel, T., Thoul, A., et al. 2007, MNRAS, 381, 1482

Chapellier, E., Le Contel, J. M., Le Contel, D., et al. 1995, A\&A, 304, 406

Cunha, K., Hubeny, I., \& Lanz, T. 2006, ApJ, 647, L143

De Ridder, J., Telting, J. H., Balona, L. A., et al. 2004, MNRAS, 351, 324

Desmet, M., Briquet, M., De Cat, P., et al. 2007, CoAst, 150, 195

Desmet, M., Briquet, M., Thoul, A., et al. 2009, MNRAS, submitted

Dupret, M.-A., De Ridder, J., De Cat, P., et al. 2003, A\&A, 398, 677

Dupret, M.-A., Thoul, A., Scuflaire, R., et al. 2004, A\&A, 415, 251

Dziembowski, W. A., \& Jerzykiewicz, M. 1996, A\&A, 306, 436

Dziembowski, W. A., \& Jerzykiewicz, M. 1999, A\&A, 341, 480

Dziembowski, W. A., \& Pamyatnykh, A. A. 2008, MNRAS, 385, 2061

Handler, G., Shobbrook, R. R., Jerzykiewicz, M., et al. 2004, MNRAS, 347, 454

Handler, G., Shobbrook, R. R., \& Mokgwetsi, T. 2005, MNRAS, 362, 612

Handler, G., Jerzykiewicz, M., Rodriguez, E., et al. 2006, MNRAS, 365, 327

Jarzebowski, T., Jerzykiewicz, M., Rios Herrera, M., \& Rios Berumen, M. 1980, RM×AA, 5, 31

Jerzykiewicz, M. 1978, Acta Astronomica, 28, 465

Jerzykiewicz, M., Handler, G., Shobbrook, R. R., et al. 2005, MNRAS, 360, 619

Mathias, P., Aerts, C., Gillet, D., \& Waelkens, C. 1994, A\&A, 289, 875

Miglio, A., Montalbán, J., \& Dupret, M.-A. 2007, MNRAS, 375, L21

Opolski, A., \& Ciurla, T. 1961, Acta Astronomica, 11, 231

Pamyatnykh, A. A., Handler, G., \& Dziembowski, W. A. 2004, MNRAS, 350, 1022

Scuflaire, R., Théado, S., Montalbán, J., et al. 2008a, Ap\&SS, 316, 83

Scuflaire, R., Montalbán, J., Théado, S., et al. 2008b, Ap\&SS, 316, 149

Smith, M. A. 1980, ApJ, 240, 149

Thoul, A., Aerts, C., Dupret, M. A., et al. 2003, A\&A, 406, 287

Young, R. K. 1915, JRASC, 9, 423 